\title{
NATURAL OPERATIONS ON DIFFERENTIAL FORMS
}

\author{
BY \\ RICHARD S. PALAIS
}

1. Introduction. Suppose that from a given mathematical structure( $\left.{ }^{1}\right) S$ we have constructed in some canonical fashion two vector spaces over the same field, say $V$ and $W$. If $g$ is an automorphism( $\left.{ }^{1}\right)$ of $S$ then there will be induced by $g$ automorphisms $R_{0}^{V}$ and $R_{0}^{W}$ of $V$ and $W$ respectively, and the mappings $R^{V}: g \rightarrow R_{g}^{V}$ and $R^{W}: g \rightarrow R_{0}^{W}$ will be representations of the group $G$ of all automorphisms of $S$ in the vector spaces $V$ and $W$. Let us denote by $\mathfrak{g}(V, W)$ the space of intertwining operators for these two representations, that is to say the vector space of linear mappings $T$ of $V$ into $W$ which are equivariant in the sense that $T R_{o}^{V}=R_{o}^{W} T$ for all $g \in G$. Now in general the space of intertwining operators is the basic object of interest in studying th: relation between two representations of the same group. In the case of representations arising as above moreover the space of intertwining operators is of considerable interest in its own right, for the equivariance condition becomes in this setting what is usually referred to as a "naturality" condition, so that elements of $g(V, W)$ might well be termed the natural linear maps of $V$ into $W$. The "natural" here means relative to the way that $V$ and $W$ are constructed out of $S$ of course.

In the present paper we will solve explicitly the problem of determining the spaces $g(V, W)$ for the case that $S$ is a differentiable manifold and $V$ and $W$ are certain spaces of tensor fields on $S$. Before stating the results we introduce some notation which will be fixed for the remainder of the paper.

2. Notation. $M$ will denote a connected, $n$-dimensional differentiable $\left({ }^{2}\right)$ manifold. For some purposes $M$ will have to be compact but this assumption will always appear explicitly. The tangent space to $M$ at a point $m$ will be denoted by $M_{m}$ and we use $\left(M_{m}\right)_{s}^{r}$ to denote the space $\left(\otimes^{r} M_{m}\right) \otimes\left(\otimes^{*} M_{m}^{*}\right)$ of tensors at $m$ contravariant of rank $r$ and covariant of rank $s$. Elements of this space are multilinear functionals on the cartesian product of $r$ copies of $M_{m}^{*}$ and $s$ copies of $M_{m}$. A tensor field $T$ contravariant of rank $r$ and covariant of rank $s$ is a function on $M$ which assigns to each $m \in M$ an element $T_{m} \in\left(M_{m}\right)_{s}^{r}$. Defining differentiability for tensor fields in the customary manner, the set of differentiable tensor fields on $M$ which are contravariant of rank $r$ and covariant of rank $s$ becomes a real vector space $V_{s}^{\tau}$ under pointwise linear operations. As is usual we identify each $\left(M_{m}\right)_{0}^{0}$ with the field of real

Presented to the Society October 26, 1957; received by the editors January 31, 1958.

1 Metamathematical terms are notoriously difficult to define in a precise sense that has the proper degree of generality. Since any reasonable interpretation of these terms will be acceptable for present purposes we have not attempted to make them precise.

2 By differentiable we shall always mean of class $C^{\infty}$. 
numbers so that $V_{0}^{0}$ is the space of differentiable real valued functions on $M$. We denote by $\Im(M)$ the direct sum $\oplus_{r, s=0}^{\infty} V_{s}^{r}$.

An automorphism of $M$ is what is usually called a diffeomorphism, that is a nonsingular differentiable homeomorphism of $M$. We denote by $G$ the group of all diffeomorphisms of $M$, and by $\delta \phi$ the differential of an element $\phi$ of $G$. Thus for $m \in M \delta \phi$ maps $M_{m}$ isomorphically onto $M_{\phi(m)}$. Since each $V_{s}^{r}$ is canonically constructed from $M$, each $\phi \in G$ induces an automorphism $R_{\phi}$ of $V_{s}^{r}$. If $T \in V_{s}^{r}$ then $R_{\phi}$ is given explicitly by

$$
\begin{aligned}
R_{\phi}(T)_{m}\left(\theta_{1} \cdots \theta_{r} ; v_{1}\right. & \left.\cdots v_{s}\right) \\
& =T_{\phi^{-1}(m)}\left(\delta \phi^{*}\left(\theta_{1}\right) \cdots \delta \phi^{*}\left(\theta_{r}\right) ; \delta \phi^{-1}\left(v_{1}\right) \cdots \delta \phi^{-1}\left(v_{\varepsilon}\right)\right) .
\end{aligned}
$$

If $T$ is given in local coordinates $x_{1} \cdots x_{n}$ at $\phi^{-1}(m)$ by

$$
T=\sum a_{j_{1} \cdots j_{8}}^{i_{1} \cdots i_{r}} X_{i_{1}} \otimes \cdots \otimes X_{i_{r}} \otimes d x_{j_{1}} \otimes \cdots \otimes d x_{j_{\text {⿰ }}} \text { (where } X_{i}=\left(\partial / \partial x_{i}\right),
$$

then $R_{\phi}(T)$ is given near $m$ by:

$\sum a_{j_{1} \cdots j_{\ell}}^{i_{1} \cdots i_{r}} \circ \phi^{-1} \delta \phi\left(X_{i_{1}}\right) \otimes \cdots \otimes \delta \phi\left(X_{i_{r}}\right) \otimes d\left(x_{j_{1}} \circ \phi^{-1}\right) \otimes \cdots \otimes d\left(x_{j_{r}} \circ \phi^{-1}\right)$,

a formula which we will have occasion to refer to several times during explicit computations.

These representations $R: \phi \rightarrow R_{\phi}$ of $G$ in each $V_{s}^{r}$ define a representation of $G$ also denoted by $R$ in $\Im(M)$. By an $M$-tensor space we mean an invariant subspace of $\Im(M)$ under this representation and we will use the already overworked symbol $R$ to denote the restricted representation of $G$ in each $M$ tensor space.

We now introduce a topology $T^{\infty}$ in $G$ which will be used only to define the notion of continuous arcs. A neighborhood base for the identity is given as follows: let $(x)=\left(x_{1} \cdots x_{n}\right)$ be a coordinate system in a coordinate neighborhood $U$ of $M$, let $K$ be a compact subset of $U$ and $\epsilon$ a positive number; then a typical element of a neighborhood base at the identity is given by $N((x), U, K, k, \boldsymbol{\epsilon})$ which consists of all $\phi \in G$ for which $\phi(K) \subseteq U$ and for which all derivatives up to order $k$ of the functions $x_{i} \circ \phi$ with respect to the $x_{j}$ are uniformly within $\epsilon$ of the corresponding derivatives of $x_{i}$ on $K$ ( $k$ is any non-negative integer and we consider the identity operator a zero order derivative). This topology makes $G$ into a topological group $\left({ }^{3}\right)$. An arc in $G$

${ }^{3}$ This can be seen by the following more intrinsic definition of $T^{\infty}$. Define $B^{k}(M)$ inductively by $B^{0}(M)=M, B^{k+1}(M)=$ the bundle of frames of $B^{k}(M)$. Then each $\phi \in G$ induces a diffeomorphism $\phi^{(k)}$ of $B^{(k)}(M)$ and $i^{(k)}: \phi \rightarrow \phi^{(k)}$ is an isomorphism of $G$ into the group $G^{(k)}$ of diffeomorphisms of $B^{(k)}(M)$. Now it is known that the compact-open topology makes $G^{(k)}$ a topological group (cf. Theorem 4 of R. Arens' Topologies for homeomorphism groups, Amer. J. Math. vol. 68 no. 4 (1946)). Carrying the compact-open topology for $G^{(k)}$ back to $G$ via $i^{(k)}$ we get a topology $T^{(k)}$ which makes $G$ a topological group. Clearly $T^{(k)} \subseteq T^{(k+1)}$ and it is easily shown that $T^{\infty}$ is the union of the $T^{(k)}$. 
relative to $T^{\infty}$ is called an isotopy. Let $G_{c}$ be the subgroup of $G$ consisting of those $\phi$ for which there exists a compact subset $K$ of $M$ such that $\phi$ maps $M-K$ identically and let $G_{0}$ be the arc component of the identity in $G_{c}$ relative to $T^{\infty}$. It is easily seen that $G_{0}$ is a normal subgroup of $G$.

If $V$ is an $M$-tensor space we will denote by $R^{0}$ the restriction to $G_{0}$ of the representation $R$ of $G$ in $V$. If $V$ and $W$ are two $M$-tensor spaces then we will denote by $g(V, W)$ the space of intertwining operators for the representations $R$ of $G$ in $V$ and $W$, and by $g^{\circ}(V, W)$ the space of intertwining operators for the representations $R^{0}$ of $G_{0}$ in $V$ and $W$. Clearly $g(V, W) \subseteq g^{0}(V, W)$. Generally speaking $g^{0}(V, W)$ may have a few interesting operators not in $\mathscr{g}(V, W)$, and it is easy to determine $\mathfrak{g}(V, W)$ once $\mathfrak{g}^{0}(V, W)$ is known. In succeeding papers we expect to investigate the general problem of determining these spaces of intertwining operators. In the present paper we solve what are perhaps the most interesting special cases; namely when $V$ and $W$ are spaces of differential forms.

3. Results. Let $\Phi^{p} \subseteq V_{p}^{0}$ be the space of differential forms of degree $p$ on $M, d^{p}: \Phi^{p} \rightarrow \Phi^{p+1}$ the exterior derivative restricted to $\Phi^{p}, Z^{p}=$ null space of $d^{p}=$ space of closed forms of degree $p$, and $B^{p}=$ image of $d^{p-1}=$ space of exact forms of degree $p$. For each of the above spaces we denote the subspace of elements with compact support by using a subscript $c$; thus $B_{c}^{p}$ is the space of exact $p$-forms with compact support. Our results are the following $\left({ }^{4}\right)$ :

$g^{0}\left(\Phi^{p}, \Phi^{q}\right)=g\left(\Phi^{p}, \Phi^{q}\right)=0$ if $0 \leqq p \leqq n, 0<q \leqq n$, and $q \neq p, p+1 ;$

$\mathscr{g}^{0}\left(\Phi^{p}, \Phi^{p}\right)=\mathscr{S}\left(\Phi^{p}, \Phi^{p}\right)=$ constant multiples of the identity map if $p>0$; $g^{0}\left(\Phi^{p}, \Phi^{p+1}\right)=g\left(\Phi^{p}, \Phi^{p+1}\right)=$ constant multiples of $d^{p}$;

if $M$ is compact then in addition

$\mathscr{g}^{0}\left(\Phi^{p}, \Phi^{0}\right)=\mathscr{g}\left(\Phi^{p}, \Phi^{0}\right)=0$ if $0<p<n$;

$\mathscr{g}^{0}\left(\Phi^{0}, \Phi^{0}\right)=\mathscr{g}\left(\Phi^{0}, \Phi^{0}\right)=$ constant multiples of the identity map;

$g^{0}\left(\Phi^{n}, \Phi^{0}\right)=$ constant multiples of $\int_{M}$ (where $\int_{M}$ is integration of an $n$-form over the fundamental cycle) if $M$ is orientable;

$=0$ if $M$ is nonorientable;

$\mathfrak{g}\left(\Phi^{n}, \Phi^{0}\right)=0$ if $M$ is nonorientable, or if $M$ is orientable and reversible $\left({ }^{5}\right)$; = constant multiples of $\int_{M}$ if $M$ is orientable and irreversible $\left(^{5}\right)$;

$\mathscr{g}^{0}\left(Z^{p}, \Phi^{q}\right)=\mathscr{g}\left(Z^{p}, \Phi^{q}\right)=0$ if $q>0$ and $p \neq q$ (this holds even if $M$ is not compact);

- A suggestion by Professor I. E. Segal that amounted to a conjecture of this fact was the motivation for the author's work on this paper. I would like to thank Professor Segal both for this and for many interesting conversations concerning the general subject matter of this paper. We note in passing that this particular result says that the exterior derivative on $p$-forms is determined to within a scalar factor by the condition that it be a linear mapping into $p+1$ forms which commutes with all diffeomorphisms. This is a considerably simpler and more intrinsic axiomatic characterization of the exterior derivative than the usual one (cf. p. 10 of Lie groups and differential geometry, K. Nomizu, Tokyo, 1956). In particular the fact that $d^{p+1} \circ d^{p}=0$ follows automatically from $\mathcal{G}\left(\Phi^{p}, \Phi^{p+2}\right)=0$.

- An orientable manifold is called reversible or irreversible according as it does or does not admit an orientation reversing diffeomorphism. 
$g^{0}\left(Z^{q}, \Phi^{q}\right)=\mathscr{g}\left(Z^{q}, \Phi^{q}\right)=$ constant multiples of the inclusion map if $q>0$ (this holds even if $M$ is not compact);

$g^{0}\left(Z^{p}, \Phi^{0}\right) \cong H_{p}(M)$, the $p$-dimensional real homology space of $M$; $\mathscr{g}\left(Z^{p}, \Phi^{0}\right) \cong N_{p}(M)$, the subspace of $H_{p}(M)$ consisting of elements which are "natural" in the sense of being left fixed by all the automorphisms of $H_{p}(M)$ induced by diffeomorphisms of $M$.

4. A remark concerning finite coverings. In this section all topological spaces are assumed to be locally connected and Hausdorff. A mapping $f: X \rightarrow Y$ is called a local homeomorphism if for each $x \in X$ a neighborhood of $x$ is mapped homeomorphically by $f$ onto a neighborhood of $f(x)$. If $X$ and $Y$ are connected and if $f$ is a mapping of $X$ onto $Y$ with the property that each $y \in Y$ has a neighborhood $V$ such that each component of $f^{-1}(V)$ is mapped homeomorphically onto $V$ by $f$, then $f$ is called a covering map. In this case an obvious and well-known connectivity argument show that the cardinal number $\nu$ of the set $f^{-1}(y)$ is the same for all $y \in Y ; \nu$ is called the number of sheets of the covering and if $\nu$ is a finite integer $n$ then $f$ is called a finite covering or more specifically an $n$-sheeted covering.

It is clear that a covering map is a local homeomorphism. The converse is false and the difference between the two concepts is rather complicated in general. However for finite coverings we have

4.1. Lemma. If $X$ and $Y$ are connected and $f: X \rightarrow Y$ is a local homeomorphism for which there is an integer $n$ such that $f^{-1}(y)$ has exactly $n$ points for each $y \in Y$ then $f$ is an $n$-sheeted covering.

Proof. Given $y \in Y$ let $f^{-1}(y)=\left\{x^{1}, \cdots, x^{n}\right\}$ and let $W^{1}, \cdots, W^{n}$ be disjoint neighborhoods of the $x^{i}$ such that $f$ maps each $W^{i}$ homeomorphically onto a neighborhood of $y$. Let $V$ be the component of $y$ in $\bigcap_{i=1}^{n} f\left(W^{i}\right)$ and let $U^{i}=$ component of $x^{i}$ in $f^{-1}(V) \cap W^{i}$. The fact that $f$ is uniformly $n$-to-one insures that $U^{1}, \cdots, U^{n}$ are all the components of $f^{-1}(V)$ and each is clearly mapped homeomorphically onto $V$. q.e.d.

It is well-known that if $X$ and $Y$ are connected and $X$ compact then a local homeomorphism of $X$ into $Y$ is a finite covering. The following generalization of this fact is perhaps also fairly well-known, but it does not seem to appear in the literature.

4.2. Theorem. Let $X$ and $Y$ be connected, locally connected, and locally compact Hausdorff spaces. A necessary and sufficient condition for a local homeomorphism $f: X \rightarrow Y$ to be a finite covering is that the inverse image by $f$ of each compact subset of $Y$ is a compact subset of $X$.

Proof. Necessity is obvious so we prove only sufficiency. Let $Y_{n}$ $=\left\{y \in Y: f^{-1}(y)\right.$ contains at least $n$ distinct points $\}$. Clearly the fact that $f$ is a local homeomorphism implies that each $Y_{n}$ is open. We show next that 
each $Y_{n}$ is closed. In fact let $\bar{y}$ be adherent to $Y_{n}$ and let $\left\{y_{\sigma}\right\}$ be a net in $Y_{n}$ approaching $\bar{y}$. Let $x_{\sigma}^{1}, \cdots, x_{\sigma}^{n}$ be distinct points in $f^{-1}\left(y_{\sigma}\right)$. By passing to a subnet of $\left\{y_{\sigma}\right\}$ if necessary we may suppose that each $\left\{x_{\sigma}^{i}\right\}$ approaches a limit $x^{i}$ in the one point compactification of $X$. It follows from the property assumed of $f$ that actually $x^{i} \in X$. It will suffice to show that the $x^{i}$ are distinct. But if $x^{i}=x^{j}=z$ with $i \neq j$ there would be distinct points $x_{\sigma}^{i}$ and $x_{\sigma}^{j}$ arbitrarily close to $z$ with $f\left(x_{\sigma}^{i}\right)=f\left(x_{\sigma}^{j}\right)=y_{\sigma}$, contradicting the local homeomorphism property of $f$ at $z$. It follows now that each $Y_{n}$ is either empty or all of $Y$. Let $y$ be in the range of $f$. The properties assumed for $f$ imply that $f^{-1}(y)$ is both discrete and compact, and hence contains only finitely many, say $n$, points. Then $y \in Y_{n}$ but $y \notin Y_{n+1}$ so $Y_{n}=Y$ and $Y_{n+1}=\varnothing$ so $Y=Y_{n}$ $-Y_{n+1}$ and by (4.1) $f$ is an $n$-sheeted covering. q.e.d.

In the following $\|x\|$ denotes the distance of a point $x$ in $R^{n}$ from the origin of $R^{n}:\|x\|^{2}=\sum_{i=1}^{n} x_{i}^{2}$. If $f: R^{n} \rightarrow R^{n}$ then a necessary and sufficient condition that $f^{-1}$ map compact sets into compact sets is clearly that $\lim _{\|x\|+\infty}\|f(x)\|$ $=\infty$. Recalling that $R^{n}$ is simply connected the implicit function theorem gives the following corollary of (4.2), which by all rights should be a very classical theorem.

4.3. Corollary. Let $f_{1}, \cdots, f_{n}$ be $n$ real valued functions of $n$ real variables. Necessary and sufficient conditions that the mapping $f: R^{n} \rightarrow R^{n}$ defined by $f(x)=\left(f_{1}(x), \cdots, f_{n}(x)\right)$ be a diffeomorphism of $R^{n}$ onto itself are:

(1) $\operatorname{det}\left(\partial f_{i} / \partial x_{j}\right)$ never vanishes.

(2) $\lim _{\|x\| \rightarrow \infty}\|f(x)\|=\infty$.

5. Extending germs of diffeomorphisms.

5.1. Lemma. Let $\psi$ be a differentiable mapping of a neighborhood of a point $m$ of $M$ into $M$ such that $\psi(m)=m$ and such that $\delta \psi$ maps $M_{m}$ identically. Then if $U$ is any neighborhood of $m$ we can find a smaller neighborhood $V$ and an isotopy $s \rightarrow \phi^{s}$ of $[0,1]$ into $G_{0}$ such that $\phi^{0}$ is the identity map of $M$, each $\phi^{8}$ maps the complement of $U$ identically, and $\phi^{1}|V=\psi| V$.

Proof. By introducing a coordinate system at $m$ this lemma becomes an immediate consequence of the following one.

5.2. Lemma. Let $f_{1} \cdots f_{n}$ be $n$ differentiable real valued functions defined in a neighborhood of the origin in $R^{n}$ such that $f_{i}(0)=0$ and $\left(\partial f_{i} / \partial x_{j}\right)_{0}=\delta_{i j}$. Then for every sufficiently small positive number $r$ there exists a differentiable mapping $F$ of $R^{n} \times[0,1]$ into $R^{n}$ with the following properties:

(1) $F^{*}: x \rightarrow F(x, s)$ is a diffeomorphism of $R^{n}$ onto itself for each $s$ in $[0,1]$.

(2) $F^{0}$ is the identity map of $R^{n}$.

(3) $F^{s}(x)=x$ if $\|x\|>2 r$ and $0 \leqq s \leqq 1$.

(4) $F^{1}(x)=\left(f_{1}(x), \cdots, f_{n}(x)\right)$ if $\|x\| \leqq r$.

(5) $s \rightarrow F^{8}$ is an isotopy. 
Proof. Let us write $f_{i}(x)=x_{i}+A_{i}(x)$. Then by the hypotheses on the $f_{i}$ it follows that the $A_{i}$ are differentiable functions defined near the origin of $R^{n}$ and that $A_{i}(0)=\left(\partial A_{i} / \partial x_{j}\right)(0)=0$. Given $r>0$ let $\sigma_{r}$ be a differentiable function of one real variable such that $\sigma_{r}(u)=1$ if $0 \leqq u \leqq r^{2}, \sigma_{r}(u)=0$ if $u \geqq 4 r^{2}$, while for all $u 0 \leqq \sigma_{r}(u) \leqq 1$ and $\left|\sigma_{r}^{\prime}(u)\right|<1 / r^{2}$. The existence of a class $C^{1}$ function with these properties is trivial, and by regularization (that is, convoluting with a $C^{\infty}$ function of integral unity and support a small neighborhood of the origin) we can replace the $C^{1}$ function by a $C^{\infty}$ one. For $r$ so small that all the $f_{i}$ are defined in the ball of radius $3 r$ about the origin we define $F: R^{n} \times[0,1] \rightarrow R^{n}$ by

$$
\begin{array}{ll}
F_{i}(x, s)=x_{i}+s \sigma_{r}\left(\|x\|^{2}\right) A_{i}(x), & \text { for }\|x\| \leqq 2 r, \\
F_{i}(x, s)=x_{i} & \text { for }\|x\| \geqq 2 r .
\end{array}
$$

Once conclusion (1) is established; the remaining conclusions are obvious. Since $F^{s}(x)=x$ for $\|x\| \geqq 2 r$ it follows from (4.3) above that the lemma will be established if we can show that provided $r$ is sufficiently small $\operatorname{det}\left(\partial F_{i}^{s} / \partial x_{j}\right)$ does not vanish in the ball $\|x\| \leqq 2 r$. Now since $\partial F_{i}^{s} / \partial x_{j}=\delta_{i j}+s a_{i j}(x, r)$ where

$$
a_{i j}(x, r)=2 \sigma_{r}^{\prime}\left(\|x\|^{2}\right) x_{j} A_{i}(x)+\sigma_{r}\left(\|x\|^{2}\right)\left(\partial A_{i} / \partial x_{j}\right)(x),
$$

it will suffice to show that $a_{i j}(x, r)$ approaches zero as $r$ goes to zero, uniformly on $\|x\| \leqq 2 r$. Now since the $A_{i}$ are differentiable functions satisfying $A_{i}(0)=\left(\partial A_{i} / \partial x_{j}\right)(0)=0$ it follows from Taylor's theorem that we can write $A_{i}(x)=\|x\|{ }^{2} B_{i}(x)$ and $\left(\partial A_{i} / \partial x_{j}\right)(x)=\|x\| B_{i j}(x)$ where the functions $\left|B_{i}\right|$ and $\left|B_{i j}\right|$ are bounded. Letting $N$ be a bound for the latter functions and recalling that $\left|\sigma_{r}^{\prime}\right|<1 / r^{2}$ and $\left|\sigma_{r}\right|<1$ we have $\left|a_{i j}(x, r)\right| \leqq N\|x\|\left(2\|x\|^{2} / r^{2}+1\right)$ so that on $\|x\| \leqq 2 r$ we have $\left|a_{i j}(x, r)\right| \leqq 9 N r$ which goes to zero uniformly in $x$ as $r$ goes to zero. The lemma is thereby proved.

Let $B(M)$ denote the bundle of frames of $M$. Each $\phi \in G$ induces a diffeomorphism $\phi^{\prime}$ of $B(M): \phi^{\prime}$ maps the frame $e=\left(e_{1}, \cdots, e_{n}\right)$ into the frame $\phi^{\prime}(e)=\left(\delta \phi\left(e_{1}\right), \cdots, \delta \phi\left(e_{n}\right)\right)$.

\subsection{Lemma. $G_{0}$ acts transitively on each component of $B(M)$.}

Proof. Since each element of $G_{0}$ is isotopic to the identity it is clear that the partitioning of $B(M)$ into orbits under $G_{0}$ is at least as fine as the partitioning of $B(M)$ into components. Since we wish to show that it is not a properly finer partitioning it will suffice to show that given $m \in M$ and a frame $e$ at $m$ the orbit of $e$ under $G_{0}$ contains a neighborhood of $e$ in $B(M)$. Introducing a coordinate system at $m$ this follows easily from the following lemma.

5.4. Lemma. Given $r>0$ there exists an $\epsilon>0$ such that given $a \in R^{n}$ and $T \in G L(R, n)$ satisfying $\left|a_{i}\right|<\epsilon$ and $\left|\delta_{i j}-T_{i j}\right|<\epsilon$ there is a differentiable mapping $F: R^{n} \times[0,1] \rightarrow R^{n}$ with the following properties: 
(1) $F^{s}: x \rightarrow F(x, s)$ is a diffeomorphism of $R^{n}$ onto itself for each $s$ in $[0,1]$;

(2) $F^{0}$ is the identity map of $R^{n}$;

(3) $F^{s}(x)=x$ if $\|x\| \geqq 2 r$;

(4) $F^{1}(x)=T x+a$ if $\|x\| \leqq r$;

(5) $s \rightarrow F^{*}$ is an isotopy.

Proof. Let $\sigma_{r}$ be a function with the properties described in the proof of (5.3). Define:

$$
F(x, s)=x+s \sigma_{r}\left(\|x\|^{2}\right)(T x-x+a) .
$$

Then as in the proof of (5.3) once (1) is demonstrated the properties (2)-(5) are obvious. Now (1) will follow from (4.3) once it is shown that $\operatorname{det}\left(\partial F_{i}^{i} / \partial x_{j}\right)$ never vanishes on $\|x\| \leqq 2 r$, provided $\epsilon$ is chosen sufficiently small. Since

$$
\begin{gathered}
\partial F_{i}^{s} / \partial x_{j}=\delta_{i j}+2 s x_{j} \sigma_{r}^{\prime}\left(\|x\|^{2}\right)\left(\sum_{j} T_{i j} x_{j}-x_{i}+a_{i}\right) \\
+s \sigma_{r}\left(\|x\|^{2}\right)\left(T_{i j}-\delta_{i j}\right) .
\end{gathered}
$$

Remembering that $\left|\sigma_{r}\right| \leqq 1$ and $\left|\sigma_{r}^{\prime}\right| \leqq 1 / r^{2}$ we see that for $0 \leqq s \leqq 1$ and $\|x\| \leqq 2 r$

$$
\left|\partial F_{i}^{8} / \partial x_{j}-\delta_{i j}\right| \leqq(4 / r)(\|T-I\| r+\epsilon)+\epsilon .
$$

Since $\|T-I\|$ can be made arbitrarily small by making $\epsilon$ small enough the proof is complete.

5.5. Theorem. Let $\psi$ be a differentiable mapping of a neighborhood of a point $m$ of $M$ into $M$, and suppose that $\delta \psi_{m}$ is nonsingular. If $M$ is orientable assume in addition that $\delta \psi_{m}$ is orientation preserving. Then there exists $a \phi$ in $G_{0}$ such that $\phi|V=\psi| V$ for some neighborhood $V$ of $m$.

Proof. Let $e$ be a frame at $m$, and let $e^{\prime}=\left(\delta \psi\left(e_{1}\right), \cdots, \delta \psi\left(e_{n}\right)\right)$, a frame at $\psi(m)$. If $M$ is nonorientable then $B(M)$ is connected. If $M$ is orientable then $B(M)$ has two components, but in this case the extra assumption on $\delta \psi_{m}$ assures that nevertheless $e$ and $e^{\prime}$ belong to the same component. By (5.3) we can find $\sigma \in G_{0}$ such that $\sigma^{\prime}\left(e^{\prime}\right)=e$. Then $\sigma \circ \psi(m)=m$ and $\delta(\sigma \circ \psi)$ maps $M_{m}$ identically so it follows from (5.1) that we can find $\theta \in G_{0}$ such that $\sigma \circ \psi|V=\theta| V$ for some neighborhood $V$ of $m$. Then clearly if we put $\phi$ $=\sigma^{-1} \circ \theta$ the conclusion of the theorem is satisfied.

A coordinate system $(x)=\left(x_{i} \cdots x_{n}\right)$ in a coordinate domain $U$ of $M$ will be said to be centered at $m \in U$ if $x_{i}(m)=0 i=1, \cdots, n$.

5.6. Corollary. Let $(x)$ and $(y)$ be coordinate systems in $M$ centered at $m_{1}$ and $m_{2}$ respectively. If $M$ is orientable assume in addition that $(x)$ and $(y)$ are similarly oriented. There exists $a \phi \in G_{0}$ such that $x_{i}\left|V=y_{i} \circ \phi\right| V, i=1, \cdots, n$. 
The theorems of this section will be needed later on when we will need to know that there exist certain diffeomorphisms in $G_{0}$ having specified properties near a given point. We will need one more theorem of this type.

5.7. Theorem. Let $r$ and $\epsilon$ be positive numbers and let $0<c<1$. Then there exists a differentiable map $F: R^{n} \times[0,1] \rightarrow R^{n}$ with the following properties:

(1) $F^{s}: x \rightarrow F(x, s)$ is a diffeomorphism of $R^{n}$ onto itself for each $s$ in $[0,1]$,

(2) $F^{0}$ is the identity map of $R^{n}$,

(3) $F^{s}(x)=x$ if $\|x\|>r+\epsilon$ and $0 \leqq s \leqq 1$,

(4) $F^{1}(x)=c x$ if $\|x\|<r$,

(5) $s \rightarrow F^{8}$ is an isotopy.

Proof. Construct as in (5.2) a differentiable function $\sigma$ of one real variable such that $\sigma(u)=1$ if $0 \leqq u \leqq r^{2}, \sigma(u)=0$ if $u>(r+\epsilon)^{2}$, and $\sigma^{\prime}(u) \leqq 0$ if $r^{2} \leqq u$ $\leqq(r+\epsilon)^{2}$. Define

$$
F(x, s)=\left(1+s(c-1) \sigma\left(\|x\|^{2}\right)\right) x .
$$

An easy calculation shows that the Jacobian matrix of $F^{s}$ is the sum of a positive multiple of the identity matrix and a positive semi-definite matrix. Hence $F^{s}$ is nonsingular at every point and by (4.3) each $F^{s}$ is a diffeomorphism of $R^{n}$ with itself. Properties (2)-(5) are then as usual easily verified.

\section{Localizable operations.}

Definition. Let $V$ and $W$ be $M$-tensor spaces. A linear transformation of $V$ into $W$ will be called localizable if whenever $v_{1}$ and $v_{2}$ in $V$ agree in a neighborhood of a point $m$ it follows that $T v_{1}$ and $T v_{2}$ also agree in a neighborhood of $m$. Equivalently $T$ is localizable if whenever $v \in V$ vanishes in a neighborhood of a point $m$ it follows that $T v$ vanishes at $m$.

6.1. TheOREM. Let $V$ and $W$ be $M$-tensor spaces and suppose $W \subseteq V_{s}^{r}$ where $r-s \neq 0$. Then $\mathfrak{g}^{0}(V, W)$ consists entirely of localizable operators.

Proof. Let $m \in M$ and suppose $v \in V$ vanishes in a neighborhood $U$ of $m$. Letting $T$ denote an arbitrary element of $g^{\circ}(V, W)$ we must show that $(T v)_{m}=0$. If $q$ is a real number sufficiently close to unity it follows directly from (5.4) that we can find $\phi \in G_{0}$ such that $\phi(m)=m, \delta \phi_{m}$ is multiplication by $q$, and $\phi$ maps the complement of $U$ identically. We note that the isomorphism of $\left(M_{m}\right)_{s}^{r}$ induced by $\delta \phi_{m}$ is $q^{r-s}$. Now since $v$ vanishes in $U$ while $\phi$ maps the complement of $U$ identically it follows that $R_{\phi} v=v$. Since $T \in \mathscr{g}^{0}(V, W)$ we get $(T v)_{m}=\left(T R_{\phi} v\right)_{m}=\left(R_{\phi} T v\right)_{m}=q^{r-8}(T v)_{m}$. Since $r-s \neq 0$ it follows by taking $q \neq 1$ that $(T v)_{m}=0$. q.e.d.

6.2. Corollary. If $q \neq 0$ then the spaces $\mathscr{S}\left(\Phi^{p}, \Phi^{q}\right), \mathfrak{I}^{0}\left(\Phi^{p}, \Phi^{q}\right), \mathscr{g}\left(Z^{p}, \Phi^{q}\right)$, and $g^{0}\left(Z^{p}, \Phi^{q}\right)$ consist entirely of localizable operators.

We note that the restriction $q \neq 0$ is essential. For example if $M$ is compact and orientable then $w \rightarrow \int_{M} w$ is a nonlocalizable element of $\mathcal{g}^{0}\left(\Phi^{n}, \Phi^{0}\right)$. 
6.3. Corollary. Let $T$ be an element of one of the spaces listed in (6.2). Let $w^{1}$ and $w^{2}$ be in the domain of $T$ and suppose there exist coordinate systems $(x)$ and $(y)$ centered at $m_{1}$ and $m_{2}$ respectively such that

$$
\begin{aligned}
w^{1} & =\sum a_{i_{1} \cdots i_{p}}(x) d x_{i_{1}} \wedge \cdots \wedge d x_{i_{p}}, \\
w^{2} & =\sum a_{i_{1} \cdots i_{p}}(y) d y_{i_{1}} \wedge \cdots \wedge d y_{i_{p}}
\end{aligned}
$$

near $m_{1}$ and $m_{2}$ respectively (the sums are over all p-tuples $\left(i_{1}, \cdots, i_{p}\right)$ such that $\left.1 \leqq i_{1}<\cdots<i_{p} \leqq n\right)$. If

$$
T w^{1}=\sum b_{j_{1} \cdots j_{q}}(x) d x_{j_{1}} \wedge \cdots \wedge d x_{j_{q}}
$$

near $m_{1}$, then

$$
T w^{2}=\sum b_{j_{1} \cdots j_{q}}(y) d y_{j_{1}} \wedge \cdots \wedge d y_{j_{q}}
$$

near $m_{2}$.

Proof. Choose $\phi \in G_{0}$ satisfying (5.6). From the formula $\left(^{*}\right)$ in $\$ 2$ it follows that $\left(R_{\phi}-w^{2}\right)_{m}=w_{m}^{1}$ for $m$ in a neighborhood of $m_{1}$. Since $T$ is localizable it follows that $\left(T w^{1}\right)_{m}=\left(T R_{\phi^{-1}} w^{2}\right)_{m}$ for $m$ in a neighborhood of $m_{1}$. Since $T$ is an intertwining operator we get $\left(T w^{1}\right)_{m}=\left(R_{\phi^{-1}} T w^{2}\right)_{m}$ for $m$ in a neighborhood of $m_{1}$. Once again using the formula $\left(^{*}\right)$ our result follows directly.

7. Basic differential forms.

Definition. Let $w \in \Phi^{q}, 0 \leqq q<n$. We say that $w$ is a basic $q$-form of the first kind at $m \in M$ if there is a coordinate system $(x)$ centered at $m$ with spherical domain $U$ such that the support of $w$ is a compact subset of $U$ and $w=f d x_{1} \wedge \cdots \wedge d x_{q}$, where $f\left|V=x_{q+1}\right| V$ for some neighborhood $V$ of $m$. We say that $w$ is a basic $q$-form of the first kind if it is such at some $m \in M$.

Definition. Let $w \in \Phi^{q}, 0 \leqq q \leqq n$. We say that $w$ is a basic $q$-form of the second kind at $m \in M$ if there is a coordinate system $(x)$ centered at $m$ with spherical domain $U$ such that the support of $w$ is a compact subset of $U$ and $w=f d x_{1} \wedge \cdots \wedge d x_{q}$ where $f$ is constant near $m$. We say that $w$ is a basic $q$-form of the second kind if it is such at some $m \in M$.

7.1. Theorem. Let $(x)$ be a coordinate system with spherical domain $U$ centered at $m \in M$. Let $w$ be a q-form with compact support included in $U$. If $0 \leqq q<n$ then $w$ is the sum of a finite number of basic q-forms of the first and second kind at $m$.

Proof. Writing $w=\sum a_{i_{1} \cdots i_{q}} d x_{i_{1}} \wedge \cdots \wedge d x_{i_{q}}$ we note that each summand on the right, after a reindexing of $(x)$, takes the form $g d x_{1} \wedge \cdots \wedge d x_{q}$ where $g$ is a differentiable function with support a compact subset of $U$, hence we may assume that $w$ has this form. Let $c$ be a positive real number so large that $\left(x_{1}, \cdots, x_{q}, x_{q+1}+g / c, x_{q+2}, \cdots, x_{n}\right)$ is a coordinate system in $U$. Let $h$ be a differentiable function which is unity on the support of $g$ and vanishes outside $U$ so that $g=g h$. Then clearly 


$$
\begin{aligned}
w= & \left(\operatorname{ch}\left(x_{q+1}+g / c-g(m) / c\right) d x_{1} \wedge \cdots \wedge d x_{q}\right) \\
& +\left(-\operatorname{ch} x_{q+1} d x_{1} \wedge \cdots \wedge d x_{q}\right)+\left(h g(m) d x_{1} \wedge \cdots \wedge d x_{q}\right) .
\end{aligned}
$$

Now the first two forms on the right are basic $q$-forms of the first kind at $m$ while the third is a basic $q$-form of the second kind at $m$ so the theorem is proved.

7.2. Corollary. Let $w \in \Phi^{q}, 0 \leqq q \leqq n$, and let $m \in M$. Then there is a neighborhood of $m$ in which w is equal to the sum of a finite number of basic $q$ forms of the first and second kinds at $m$.

Proof. First assume $q<n$ and let $U$ be a spherical coordinate domain centered at $m$. If $f$ is a differentiable function which is equal to unity on a neighborhood of $m$ and has as its support a compact subset of $U$ then $f w$ is equal to $w$ in a neighborhood of $m$ and we can apply (7.1) to fw. If $q=n$ we can find an (n-1)-form $\sigma$ and a neighborhood of $m$ in which $w=d \sigma$. We can by the first part of the proof express $\sigma$ in a neighborhood of $m$ as the sum of basic $n-1$ forms at $m$. It then suffices to note that the exterior derivative of a basic $(n-1)$-form of the first (second) kind at $m$ is a basic $n$-form of the second kind at (is zero in a neighborhood of) $m$.

7.3. Lemma. If $0 \leqq q<n$ and $w$ is a basic $q$-form of the second kind at $m$, then there are points $m^{\prime}$ arbitrarily near $m$ such that $w$ is the sum of a basic $q$ form of the first kind at $m$ and a basic $q$-form of the first kind at $m^{\prime}$.

Proof. Let $(x)$ be a coordinate system with spherical domain $U$ centered at $m$ such that the support of $w$ is a compact subset of $U$ and such that in the coordinate sphere of radius $2 r$ centered at $m$ the form $w$ is given by $c d x_{1} \wedge \cdots \wedge d x_{q}$ for some real number $c$. Let $m^{\prime}$ be the point of $U$ with coordinates $x_{i}\left(m^{\prime}\right)=0, i \neq q+1, x_{q+1}\left(m^{\prime}\right)=r$. Then

$$
w=(1 / r) x_{q+1} w+\left(1-(1 / r) x_{q+1}\right) w .
$$

Putting $y_{i}=x_{i}, i \neq q+1 ; y_{q+1}=(c / r) x_{q+1}$ and $z_{i}=x_{i}, i \neq q+1 ; z_{q+1}$ $=c\left(1-(1 / r) x_{q+1}\right)$ it is clear that $(y)$ and $(z)$ are coordinate systems with do. main $U$ centered at $m$ and $m^{\prime}$ respectively provided $c \neq 0$. Moreover near $m$ we have $(1 / r) x_{q+1} w=y_{q+1} d y_{1} \wedge \cdots \wedge d y_{q}$, while near $m^{\prime}$ we have

$$
\left(1-(1 / r) x_{q+1}\right) w=z_{q+1} d z_{1} \wedge \cdots \wedge d z_{q} .
$$

If $c=0$ let $\sigma$ be any basic $q$-form of the first kind at $m$ with support in $U$. Then $w=(w-\sigma)+\sigma$ and both terms on the right are basic $q$-forms of the first kind at $m$.

7.4. ThEOREM. If $w \in \Phi_{c}^{e}, 0 \leqq q<n$ then $w$ is the sum of a finite number of basic q-forms of the first kind.

Proof. Because of (7.3) it will suffice to show that $w$ is the sum of a finite 
number of basic $q$-forms of the first and second kinds. Let $K$ be the support of $w$ so by assumption $K$ is compact. Let $\left\{U_{1}, \cdots, U_{k}\right\}$ be a covering of $K$ by spherical coordinate neighborhoods, and let $f_{1}, \cdots, f_{k+1}$ be a differentiable partition of unity subordinate to $\left\{U_{1}, \cdots, U_{k}, M-K\right\}$ so that for $i \leqq k$, $f_{i}$ is a differentiable function with support in $U_{i}$ and $f_{1}+\cdots+f_{k}=1$ identically in $K$. Then $w=f_{1} w+\cdots+f_{k} w$ and we can apply (7.1) to each of the $f_{i} w$.

7.5. Lemma. If $w$ is a basic q-form of the first kind then there exists $\phi \in G_{0}$ such that for every sufficiently small positive real number $c$ we have $w+c R_{\phi} w$ $=R_{\psi} w$ for some $\psi \in G_{0}$.

Proof. Let $U$ be the spherical domain of a coordinate system $(x)$ centered at $m$ such that $w=f d x_{1} \wedge \cdots \wedge d x_{q}$ where the support of $f$ is included in the coordinate sphere of radius $L$ about $m$ and $f=x_{q+1}$ in the sphere of radius $r$ about $m$. By (5.7) we can find $\phi \in G_{0}$ such that $x_{i} \circ \phi=(r / L) x_{i}$ within the sphere of radius $L$ while $\phi$ maps $M-U$ identically. We note that $R_{\phi} w$ $=(L / r)^{q}\left(f \circ \phi^{-1}\right) d x_{1} \wedge \cdots \wedge d x_{q}=g d x_{1} \wedge \cdots \wedge d x_{q}$ where the support of $g$ is included in the sphere of radius $r$ about $m$. Now let $\psi^{c}$ be the map of $M$ onto itself defined by $x_{i} \circ \psi^{c}=x_{i}, i \neq q+1$ and $x_{q+1} \circ \psi^{c}=x_{q+1}+c g$ in $U$ and by $\psi^{c}\left(m^{\prime}\right)=m^{\prime}$ for $m^{\prime} \in M-U$. It is clear that each $\psi^{c}$ is differentiable and also that for sufficiently small $c$ that $\psi^{c}$ is nonsingular. It is then immediate from (4.2) and the implicit function theorem that for such small $c$ the $\psi^{c}$ are all diffeomorphisms of $M$. Moreover letting $c \rightarrow 0$ we get an isotopy of these $\psi^{c}$ with the identity map of $M$ so each of these $\psi^{c}$ is contained in $G_{0}$. From the formula $\left({ }^{*}\right)$ of $\S 2$ we see that if we denote the inverse of $\psi^{c}$ by $\psi$ then $R_{\psi} w$ $=w+c R_{\phi} w$. q.e.d.

The following innocuous looking result is actually the basic fact behind a number of our more difficult theorems.

7.6. ThEOREM. If $w \in \Phi_{c}^{a} 0 \leqq q<n$ then $w$ is a finite sum of $q$-forms of the form $\sigma-R_{g} \sigma$ where $\sigma \in \Phi_{c}^{q}$ and $g \in G_{0}$.

Proof. Because of (7.4) we can assume that $w$ is a basic $q$-form of the first kind. Then by (7.5) we can find $c>0$ and $\phi, \psi \in G_{0}$ such that $w+c R_{\phi} w=R_{\psi} w$. Then putting $g=\phi^{-1} \psi \phi$ and $\sigma=R_{\phi-1}((1 / c) w)$ we get $w=\sigma-R_{g} \sigma$. q.e.d.

8. Invariant linear functionals. The space $\Phi^{0}$ of differentiable functions on $M$ contains as an invariant one dimensional subspace the constant functions. Since $M$ is connected this space is just $Z^{0}$. We will identify $Z^{0}$ with the field of real numbers so that a linear map of a real vector space into $Z^{0}$ is to be regarded as a linear functional. We note that if $c \in Z^{0}$ and $\phi \in G$ then $R_{\phi} c=c$; in other words $R$ induces the identity representation of $G$ on $Z^{0}$. It follows that if $V$ is any $M$-tensor space then $g\left(V, Z^{0}\right)$ is just the space of $G$ invariant linear functionals on $V$, i.e. linear functionals $L$ on $V$ such that $L \circ R_{\phi}=L$ for all $\phi \in G$. Similarly $g^{0}\left(V, Z^{0}\right)$ is the space of $G_{0}$-invariant linear 
functionals on $V$. Since $Z^{0}$ is a subspace of $\Phi^{0}$ it is clear that $\mathscr{g}\left(V, Z^{0}\right)$ is a subspace of $\mathscr{g}\left(V, \Phi^{0}\right)$ and that $\mathscr{g}^{0}\left(V, Z^{0}\right)$ is a subspace of $g^{0}\left(V, \Phi^{0}\right)$. In fact since $d=d^{0} \in \mathfrak{g}\left(\Phi^{0}, \Phi^{1}\right)$ we have:

8.1. Scholium. If $V$ is any $M$-tensor space and $T \in \mathfrak{g}\left(V, \Phi^{0}\right)$ (respectively, $T \in \mathscr{g}^{0}\left(V, \Phi^{0}\right)$ ) then $d \circ T \in \mathscr{I}\left(V, \Phi^{1}\right)$ (respectively, $\left.d \circ T \in \mathscr{g}^{0}\left(V, \Phi^{1}\right)\right)$, and $T \in \mathscr{g}\left(V, Z^{0}\right)$ (respectively, $T \in \mathfrak{g}^{0}\left(V, Z^{0}\right)$ ) if and only if $d \circ T=0$.

Using the above fact we will be able to show that in a number of cases $g\left(V, \Phi^{0}\right)=g\left(V, Z^{0}\right)$. It is clear for example that this will always be the case when $\mathscr{g}\left(V, \Phi^{1}\right)=0$.

It seems very unlikely to the author that there could ever exist $G$ invariant linear functionals on $\Phi^{q}$ if $q<n$ (if $M$ is compact, orientable, and irreversible $\left({ }^{5}\right)$ then $\int_{M}$ is a $G$-invariant linear functional on $\Phi^{n}$ ), however all we can prove is the following somewhat weaker theorem.

Definition. If $V$ is a subspace of $\Phi^{q}$ then we denote by $V^{0}$ the annihilator of $V$ in $\left(\Phi^{q}\right)^{*}$, i.e. the set of linear functionals on $\Phi^{q}$ which vanish identically on $V$.

\subsection{Theorem. If $q<n$ then $g^{0}\left(\Phi^{q}, Z^{0}\right)=\left(\Phi_{c}^{q}\right)^{0}$.}

Proof. Let $L \in\left(\Phi_{c}^{q}\right)^{0}, w \in \Phi^{q}$, and $\phi \in G_{0}$. Since $\phi$ maps the complement of some compact set $K$ identically it is clear that $R_{\phi} w-w$ has its support in $K$ and hence belongs to $\Phi_{c}^{q}$. But this implies that $L\left(R_{\phi} w-w\right)=0$ so $L \in g^{0}\left(\Phi^{q}, Z^{0}\right)$. That conversely every $G_{0}$-invariant linear functional on $\Phi^{a}$ must vanish on $\Phi_{c}^{a}$ is an immediate consequence of (7.6). q.e.d.

8.3. Corollary. If $M$ is compact and $q<n$, then $g^{0}\left(\Phi^{q}, Z^{0}\right)=0$ and $a$ fortiori $g\left(\Phi^{q}, Z^{0}\right)=0$.

\subsection{Corollary. If $q<n$, then $\mathfrak{g}\left(\Phi^{q}, Z^{0}\right) \subseteq\left(\Phi_{c}^{q}\right)^{0}$.}

Perhaps the deepest result of this paper is contained in (8.3). In particular (8.3) states that there are no nontrivial linear functionals on the space of differentiable functions on $M$ which are invariant under all diffeomorphisms, provided $M$ is compact. This raises the following interesting question: if $M$ is compact and $C(M)$ is the space of all continuous real valued functions on $M$ can there exist linear functionals on $C(M)$ which are invariant under the group of all homeomorphisms of $M$ ? It follows from (8.3) that any such linear functional must vanish on all functions which are differentiable in any differentiable structure for $M$ and more generally on any function which can be written as a sum of functions each of which is equivalent to a differentiable function under a homeomorphism, but this does not completely settle the problem.

9. The effect of localizable elements of $g^{0}\left(\Phi^{p}, \Phi^{q}\right)$ on basic forms.

9.1. Theorem. Let $T$ be a localizable element of $g^{0}\left(\Phi^{p}, \Phi^{q}\right)$ and let w be a basic $p$-form of the first kind at $m \in M$. Then:

(a) Tw vanishes near $m$ unless $q=p$ or $q=p+1$. 
(b) If $q=p$ then Tw is a constant multiple of $w$ near $m$. The constant depends on $T$ but not on w or $m$.

(c) If $q=p+1$ then Tw is a constant multiple of $d^{p} w$ near $m$. The constant depends on $T$ but not on $w$ or $m$.

Proof. Let $(x)$ be a spherical coordinate system centered at $m$ such that $w=x_{p+1} d x_{1} \wedge \cdots \wedge d x_{p}$ in a neighborhood of $m$. If $a_{1}, \cdots, a_{n}$ are sufficiently small real numbers then by (5.4) we can find $\phi \in G_{0}$ such that $x_{i} \circ \phi=x_{i}+a_{i}$ near $m$. Now by the formula $\left({ }^{*}\right)$ of $\S 2$ if

then

$$
T w=\sum a_{i_{1} \cdots i_{q}} d x_{i_{1}} \wedge \cdots \wedge d x_{i_{q}}
$$

$$
R_{\phi} T w=\sum a_{i_{1} \cdots i_{q}} \circ \phi^{-1} d x_{i_{1}} \wedge \cdots \wedge d x_{i_{q}} .
$$

If $a_{p+1}=0$ then clearly $R_{\phi} w=w$ near $m$ so, since $T$ is localizable, $T w=T R_{\phi} w$ $=R_{\phi} T w$ near $m$, and by the above formula it follows that the $a_{i_{1}} \cdots i_{q}$ are functions of $x_{p+1}$ alone near $m$. Appealing to (5.4) again given real numbers $b$ and $c$ sufficiently close to unity we can find $\psi \in G_{0}$ such that near $m$ we have $x_{i} \circ \psi=b x_{i}, i \leqq p, x_{i} \circ \psi=c x_{i}, i \geqq p+2$, and $x_{p+1} \circ \psi=x_{p+1}$. Using $\left({ }^{*}\right)$ and the fact that the $a_{i_{1}} \ldots i_{q}$ depend only on $x_{p+1}$ near $m$ we see that near $m$ we have

$$
R_{\psi} T w=\sum b^{J\left(i_{1} \cdots i_{q}\right)} c^{K\left(i_{1} \cdots i_{q}\right)} c^{K\left(i_{1} \cdots i_{q}\right)} a_{i_{1} \cdots i_{q}} d x_{i_{1}} \wedge \cdots \wedge d x_{i_{q}},
$$

where $J\left(i_{1} \cdots i_{q}\right)$ is the number of the indices $i_{1} \cdots i_{q}$ which are less than or equal to $p$, and $K\left(i_{1} \cdots i_{q}\right)$ is the number of them greater than or equal to $p+2$. On the other hand it is clear that $R_{\psi} w=b^{p} w$ near $m$, so by the localizability of $T$ we have near $m$

$$
R_{\psi} T w=T R_{\psi} w=b^{p} T w=\sum b^{p} a_{i_{1} \cdots i_{q}} d x_{i_{1}} \wedge \cdots \wedge d x_{i_{q}} .
$$

Comparing these two expressions for $R_{\psi} T w$ it follows that $a_{i_{1}} \ldots i_{q}$ vanishes near $m$ unless exactly $p$ of the indices are less than or equal to $p$ and none are greater than $p+1$. Conclusion (a) of the theorem is now immediate. Moreover if $q=p$ we see that $T w=f\left(x_{p+1}\right) d x_{1} \wedge \cdots \wedge d x_{p}$ near $m$. If $r$ is sufficiently close to unity then as above we can find $\sigma \in G_{0}$ such that near $m x_{p+1} \circ \sigma^{-1}$ $=r x_{p+1}$ and $x_{i} \circ \sigma^{-1}=x_{i}$ if $i \neq p+1$. Then by $\left({ }^{*}\right)$

$$
f\left(r x_{p+1}\right) d x_{1} \wedge \cdots \wedge d x_{p}=R_{\sigma} T w=T R_{\sigma} w=r f\left(x_{p+1}\right) d x_{1} \wedge \cdots \wedge d x_{p},
$$

from which it follows that $f\left(x_{p+1}\right)=k x_{p+1}$ near $m$ for some constant $k$. That $k$ depends only on $T$ and not on $w$ or $m$ follows from (6.3). This completes the proof of conclusion (b). Finally if $q=p+1$ then the above restrictions on the $a_{i_{1}} \cdots_{i_{q}}$ imply that $T w=g\left(x_{p+1}\right) d x_{1} \wedge \cdots \wedge d x_{p+1}$. Letting $\sigma$ be as above $r g\left(r x_{p+1}\right) d x_{1} \wedge \cdots \wedge d x_{p+1}=R_{\sigma} T w=T R_{\sigma} w=r g\left(x_{p+1}\right) d x_{1} \wedge \cdots \wedge d x_{p-1}$.

This clearly implies that $g$ is constant near $m$, and just as above we see that the constant depends only on $T$. This completes the proof of conclusion (c) and of the theorem. 
9.2. Theorem. Let $T$ be a localizable element of $\mathfrak{g}^{0}\left(\Phi^{p}, \Phi^{a}\right)$ and let w be a basic p-form of the second kind at $m \in M$. Then

(a) Tw vanishes near $m$ unless $q=p$.

(b) If $q=p$ then near $m$ Tw is a constant multiple of $w$. The constant depends on $T$ but not on $w$ or $m$.

Proof. Since the proof follows along lines very close to those of the preceding theorem we mention only the necessary changes. Let $(x)$ be a spherical coordinate system centered at $m$ such that $w=a d x_{1} \wedge \cdots \wedge d x_{p}$ in a neighborhood of $m$ where $a$ is a constant. The restriction $a_{p+1}=0$ in the definition of $\phi$ is no longer necessary to insure that $R_{\phi} w=w$ near $m$. As a result it follows that the $a_{i_{1}} \cdots i_{q}$ are constant near $m$. In the definition of $\psi$ we demand that $x_{p+1} \circ \psi=c x_{p+1}$ near $m$ rather than $x_{p+1} \circ \psi=x_{p+1}$ near $m$ as before. We then have $R_{\psi} w=b^{p} w$ near $m$ as before and there is an obvious change in $R_{\psi} T w$ which implies that $a_{i_{1}} \ldots i_{q}$ vanishes near $m$ unless $i_{j}=j$. The conclusions of the theorem are then immediate.

9.3. Theorem. Let $p$ and $q$ be positive integers and let $T \in g^{0}\left(Z^{p}, \Phi^{q}\right)$. If $w$ is a basic $p$-form of the second kind at $m \in M$ and if $w \in Z^{p}$ then

(a) Tw vanishes near $m$ if $q \neq p$,

(b) If $q=p$ then $T w$ is a constant multiple of $w$ near $m$. The constant depends on $T$ but not on w or $m$.

Proof. Since $q>0$ it follows from (6.2) that $T$ is localizable. The proof continues exactly as the proof of $(9.2)$.

10. The spaces $\mathscr{I}^{0}\left(\Phi^{p}, \Phi^{q}\right)$ and $\mathscr{S}\left(\Phi^{p}, \Phi^{q}\right)$ when $q>0$.

10.1. Theorem. $g^{0}\left(\Phi^{p}, \Phi^{q}\right)=0$ provided $q \neq p, p+1$, or 0 .

Proof. Suppose that $T \in \mathcal{g}^{0}\left(\Phi^{p}, \Phi^{q}\right), w \in \Phi^{p}$, and $m \in M$. With the given restriction on $q$ we must show that $(T w)_{m}=0$. By (6.2) $T$ is localizable, so by (7.2) it suffices to consider the case where $w$ is a basic $p$-form at $m$. But then (9.1a) and (9.2a) complete the proof.

10.2. Corollary. $\mathscr{g}\left(\Phi^{p}, \Phi^{q}\right)=0$ provided $q \neq p, p+1$, or 0 .

10.3. Theorem. $\mathscr{g}^{0}\left(\Phi^{p}, \Phi^{p}\right)$ consists of the constant multiples of the identity mapping provided $p \neq 0$.

Proof. Let $T \in g^{0}\left(\Phi^{p}, \Phi^{p}\right)$. Since $T$ is localizable by (6.2) it follows from (7.2) that it will be enough to show that there is a constant $c$ such that $(T w)_{m}=c w_{m}$ whenever $w$ is a basic $p$-form at $m$. Now by $(9.1 \mathrm{~b})$ there is a constant $a$ such that for any basic $p$-form of the first kind at $m$, say $\theta, T \theta=a \theta$ in a neighborhood of $m$. Similarly by (9.2b) there is a constant $b$ such that if $\theta$ is a basic $p$-form at $m$ of the second kind then $T \theta=b \theta$ in a neighborhood of $m$. We will complete the proof by showing that $a=b$. Let $m \in M$ and let $w$ be a basic $p$-form of the second kind at $m$ with $w_{m} \neq 0$. Let $V$ be neighborhood 
of $m$ in which $w$ does not vanish and $T w=b w$. By (7.3) we can find $m^{\prime} \in V$ such that $w$ is the sum of a basic $p$-form of the first kind $\theta$ at $m$ and a basic $p$-form of the first kind $\sigma$ at $m^{\prime}$. Then $b w_{m^{\prime}}=(T w)_{m^{\prime}}=(T \theta)_{m^{\prime}}+(T \sigma)_{m^{\prime}}=a \theta_{m^{\prime}}$ $+a \sigma_{m^{\prime}}=a w_{m^{\prime}}$ from which it follows that $a=b$.

10.4. CoROllaRy. $\mathscr{g}\left(\Phi^{p}, \Phi^{q}\right)$ consists of the constant multiples of the identity mapping provided $p \neq 0$.

10.5. TheOREM. $g^{0}\left(\Phi^{p}, \Phi^{p+1}\right)$ consists of the constant multiples of $d^{p}$.

Proof. Let $T \in g^{0}\left(\Phi^{p}, \Phi^{p+1}\right)$ so that $T$ is localizable by (6.2). By (9.1c) there is a real number $c$ such that if $w$ is a basic $p$-form of the first kind at $m$ then $T w=c\left(d^{p} w\right)$ near $m$. If $w$ is a basic $p$-form of the second kind at $m$ then clearly $d^{p} w$ vanishes near $m$ and by $(9.2 \mathrm{~b})$ so does $T w$. It then follows from (7.2) that if $w$ is any $p$-form then $(T w)_{m}=c\left(d^{p} w\right)_{m}$ for each $m \in M$. q.e.d.

11. The spaces $\mathscr{g}^{0}\left(\Phi^{p}, \Phi^{0}\right)$ and $\mathscr{g}\left(\Phi^{p}, \Phi^{0}\right)$.

11.1. Lemma. If $p>0$ then $\mathscr{S}^{0}\left(\Phi^{p}, \Phi^{0}\right)=\mathscr{g}^{0}\left(\Phi^{p}, Z^{0}\right)$ and $\mathscr{g}\left(\Phi^{p}, \Phi^{0}\right)=\mathscr{g}\left(\Phi^{p}, Z^{0}\right)$.

Proof. We will prove only the first equality since it plainly implies the second. If $p>1$ then the desired result follows directly from (8.1) and (10.1), so we assume that $p=1$. Now by (8.1) and (10.3) if $T \in g^{0}\left(\Phi^{1}, \Phi^{0}\right)$ then $d \circ T=c I$ where $I$ is the identity map of $\Phi^{1}$ and $c$ is some constant. If $c=0$ then by (8.1) $T \in \mathscr{g}^{0}\left(\Phi^{p}, Z^{0}\right)$ as desired, so assume that $c \neq 0$. Then for each one form $w$ on $M$ we get $w=d((1 / c) T w)$, i.e. every one form on $M$ is derived. But if this is the case then $\Phi^{1}=Z^{1}$ and the lemma follows as a special case of (13.1) which will be proved shortly. q.e.d.

11.2. Lemma. $\mathfrak{g}\left(\Phi^{0}, \Phi^{0}\right)=[I] \oplus \mathscr{g}\left(\Phi^{0}, Z^{0}\right)$ and $\mathscr{g}^{0}\left(\Phi^{0}, \Phi^{0}\right)=[I] \oplus \mathscr{g}^{0}\left(\Phi^{0}, Z^{0}\right)$ where $[I]$ is the one-dimensional space spanned by the identity map I of $\Phi^{0}$.

Proof. We prove the second equality since it implies the first. Since $[I]$ and $g^{0}\left(\Phi^{0}, Z^{0}\right)$ are clearly disjoint subspaces of $g^{0}\left(\Phi^{0}, \Phi^{0}\right)$ it will suffice to show that any $T \in \mathscr{g}^{0}\left(\Phi^{0}, \Phi^{0}\right)$ can be written as $L+c I$ where $L \in \mathscr{g}^{0}\left(\Phi^{0}, Z^{0}\right)$ and $c$ is some real number. For this it will in turn suffice, because of (8.1), to show that we have $d(T-c I)=0$, or $d \circ T=c d$ for some real number $c$. But since $d \circ T \in \mathcal{g}^{0}\left(\Phi^{0}, \Phi^{1}\right)$ the latter is an immediate consequence of (10.5).

11.3. Theorem. If $1 \leqq p<n$ every element of $g^{0}\left(\Phi^{p}, \Phi^{0}\right)$ vanishes on $\Phi_{c}^{p}$, while the restriction of any element of $g^{0}\left(\Phi^{0}, \Phi^{0}\right)$ to $\Phi_{c}^{0}$ is a constant multiple of the identity map. In particular if $M$ is compact then $g^{0}\left(\Phi^{p}, \Phi^{0}\right)=\mathscr{g}\left(\Phi^{p}, \Phi^{0}\right)=0$ when $1 \leqq p<n$ while $\mathcal{g}^{0}\left(\Phi^{0}, \Phi^{0}\right)$ and $\mathcal{G}\left(\Phi^{0}, \Phi^{0}\right)$ consist of constant multiples of the identity mapping.

Proof. Immediate from (11.1), (11.2), and (8.2).

Among the $\mathscr{I}^{0}\left(\Phi^{p}, \Phi^{q}\right)$ and $\mathscr{G}\left(\Phi^{p}, \Phi^{q}\right)$ only $\mathscr{I}^{0}\left(\Phi^{n}, \Phi^{0}\right)$ and $\mathscr{g}\left(\Phi^{n}, \Phi^{0}\right)$ are now unaccounted for. Since $\Phi^{n}=Z^{n}$ the nature of these spaces can easily be read off from the results of $\S 13$ and we state the results here without proof. 
11.4. TheOREM. If $M$ is compact and orientable then $g^{0}\left(\Phi^{n}, \Phi^{0}\right)$ consists of constant multiples of $\int_{M}$. If $M$ is compact and nonorientable then $g^{0}\left(\Phi^{n}, \Phi^{0}\right)=0$. If $M$ is compact, orientable, and irreversible then $g\left(\Phi^{n}, \Phi^{0}\right)$ consists of constant multiples of $\int_{M}$. If $M$ is compact and nonorientable or compact, orientable, and reversible then $\mathscr{G}\left(\Phi^{n}, \Phi^{0}\right)=0$.

12. The spaces $\mathfrak{g}^{0}\left(Z^{p}, \Phi^{q}\right)$ and $\mathscr{g}\left(Z^{p}, \Phi^{q}\right)$ when $q>0$.

12.1. Theorem. If $q>0$ then $\mathscr{g}^{0}\left(Z^{q}, \Phi^{q}\right)$ and $\mathfrak{g}\left(Z^{q}, \Phi^{q}\right)$ consist of constant multiples of the inclusion mapping while for $p \neq q g^{0}\left(Z^{p}, \Phi^{q}\right)=g\left(Z^{p}, \Phi^{q}\right)=0$.

Proof. We can assume that $p>0$ since the case $p=0$ is trivial. Let $w \in Z^{p}$ and $m \in M$. By the Poincaré lemma we can find $\theta \in \Phi^{p-1}$ such that $w=d^{p-1} \theta$ in a neighborhood of $m$. Since the exterior derivative of a basic $p-1$ form of the first kind at $m$ is a basic $p$-form of the second kind at $m$, while the exterior derivative of a basic $p-1$ form of the second kind at $m$ vanishes near $m$ it follows that, near $m, w$ is the sum of a finite number of derived basic $p$-forms of the second kind at $m$. Now by (6.2) elements of $g^{0}\left(Z^{p}, \Phi^{q}\right)$ are localizable so the theorem is immediate from (9.3).

13. The spaces $g^{0}\left(Z^{p}, \Phi^{0}\right)$ and $g\left(Z^{p}, \Phi^{0}\right)$.

\subsection{Lemma. If $T \in g^{0}\left(Z^{1}, \Phi^{0}\right)$ then $d \circ T=0$.}

Proof. Since $d \circ T \in \mathfrak{g}^{0}\left(Z^{1}, \Phi^{1}\right)$ we at least have $d \circ T=c I$ by (12.1). It remains to show that in fact $c=0$. Now $T \circ d \in g^{0}\left(\Phi^{0}, \Phi^{0}\right)$ and so by (11.3) restricted to $\Phi_{c}^{0}$ it is a constant multiple of the identity, $k I$. If $f$ is a differentiable function with compact support $k(d f)=d(k f)=d(T(d f))=d \circ T(d f)$ $=c(d f)$, so $k=c$. Now let $(x)$ be a coordinate system centered at $m \in M$. If $a$ is a positive number sufficiently close to unity then by (5.7) we can find $\phi \in G_{0}$ such that in a neighborhood $V$ of $m$ we have $x_{i} \circ \phi=(1 / a) x_{i}$. Let $f$ be a differentiable real valued function with support a compact subset of $V$. Then $R_{\phi}(d f)=a(d f)$ so

$$
a c f=a(T(d f))=T(a(d f))=T\left(R_{\phi}(d f)\right)=R_{\phi}(T(d f))=c\left(R_{\phi} f\right) .
$$

If $c \neq 0$ we would get $R_{\phi} f=a f$. But since $a$ need not be equal to unity we would then get an immediate contradiction by taking the supremum of both sides of this equation. It follows that $c=0$ and the lemma is proved.

\subsection{Lemma. $\mathfrak{g}^{0}\left(Z^{p}, \Phi^{0}\right)=g^{0}\left(Z^{p}, Z^{0}\right)$.}

Proof. Let $T \in g^{0}\left(Z^{p}, \Phi^{0}\right)$. Then $d \circ T \in g^{0}\left(Z^{p}, \Phi^{1}\right)$ hence if $p \neq 1$ then $d \circ T=0$ by (12.1), while if $p=1$ then $d \circ T=0$ by (13.1). The lemma now follows from (8.1).

In what follows given a subspace $V$ of $Z^{p}$ we will denote by $V^{0}$ its annihilator in $\left(Z^{p}\right)^{*}$, i.e. the set of linear functionals on $Z^{p}$ which vanish identically on $V$. We recall that $V^{0}$ is canonically isomorphic to $\left(Z^{p} / V\right)^{*}$. 
13.3. Lemma. $\mathscr{g}^{0}\left(Z^{p}, \Phi^{0}\right) \subseteq\left(d^{p-1} \Phi_{c}^{p-1}\right)^{0}$. Hence if $M$ is compact then $g^{0}\left(Z^{p}, \Phi^{0}\right)$ $\subseteq\left(B^{p}\right)^{0}$.

Proof. If $p=0$ there is nothing to prove. If $p>0$ then in any case by (13.2) any element $T$ of $\mathscr{g}^{0}\left(Z^{p}, \Phi^{0}\right)$ is a linear functional on $Z^{p}$ so it will suffice to show that $T \circ d^{p-1}$ vanishes on $\Phi_{c}^{p-1}$. Now if $p=1$ then the proof of (13.1) gives the desired result while if $p>1$ then since $T \circ d^{p-1} \in g^{0}\left(\Phi^{p-1}, \Phi^{0}\right)$ the proof is completed by (11.3).

13.4. Theorem. If $M$ is compact then $g^{0}\left(Z^{p}, \Phi^{0}\right)$ is isomorphic to $H_{p}(M)$, the real p-dimensional homology space of $M$. In the isomorphism, which is natural, $g\left(Z^{p}, \Phi^{0}\right)$ is carried into $N_{p}(M)$, the subspace of $H_{p}(M)$ consisting of homology classes which are natural in the sense that they are left fixed by all the isomorphisms $\phi_{*}$ of $H_{p}(M)$ induced by diffeomorphisms $\phi$ of $M$.

Proof. On the one hand we have the canonical isomorphism $\left(B^{p}\right)^{0}$ $\simeq\left(Z^{p} / B^{p}\right)^{*}$, and on the other hand the de Rham isomorphism $Z^{p} / B^{p}$ $\simeq H_{p}(M)^{*}$. Since $M$ is compact, $H_{p}(M)$ is finite dimensional and therefore is reflexive. Hence we get the chain of isomorphisms

$$
H_{p}(M) \simeq H_{p}(M)^{* *} \simeq\left(Z^{p} / B^{p}\right)^{*} \simeq\left(B^{p}\right)^{0} .
$$

Tracing through these isomorphisms given $\left[c_{p}\right] \in H_{p}(M)$ we get an element $L_{\left[c_{p}\right]}$ of $\left(B^{p}\right)^{0}$ by defining $L_{\left[c_{p}\right]}(w)=\int_{c_{p}} w$ for all $w \in Z^{p}$. Every element of $\left(B^{p}\right)^{0}$ arises in this way from a uniquely determined homology class $\left[c_{p}\right]$. In particular every element of $g^{0}\left(Z^{p}, \Phi^{0}\right)$ arises in this way by (13.3). We now show that conversely everything arising in this way is an element of $g^{0}\left(Z^{p}, \Phi^{0}\right)$. If $\phi \in G$ then

$$
L_{\left[c_{p}\right]}\left(R_{\phi} w\right)=\int_{c_{p}} \phi^{*} w=\int_{\phi_{*} c_{p}} w=L_{\left[\phi_{*} c_{p}\right]}(w)=L_{\phi_{*}\left[c_{p}\right]}(w)
$$

so $L_{\left[c_{p}\right]} \circ R_{\phi}=L_{\phi_{*}\left[c_{p}\right]}$. Now if $\phi \in G_{0}$ then $\phi$ is isotopic, and a fortiori homotopic to the identity map of $M$ and $\phi_{*}$ is therefore the identity mapping of $H_{p}(M)$, so $L_{\left[c_{p}\right]} \circ R_{\phi}=L_{\left[c_{p}\right]}$ for all $\phi \in G_{0}$. This shows that $L_{\left[c_{p}\right]}$ is always in $\mathscr{g}^{0}\left(Z^{p}, \Phi^{0}\right)$ and at the same time we see that $L_{\left[c_{p}\right]}$ is in $g\left(Z^{p}, \Phi^{0}\right)$ if and only if $\phi_{*}\left[c_{p}\right]=c_{p}$ for all $\phi \in G$. q.e.d.

If $M$ is not compact then essentially the same arguments as above allow us to compute $g^{0}\left(Z_{c}^{p}, \Phi^{0}\right)$. The result is that this space is isomorphic to the dual of the $p$-dimensional cohomology space of $M$ with compact support. This in turn is known to be naturally isomorphic to the $p$-dimensional locally finite homology of $M$.

University of Chicago, Chicago, Ill. 